\title{
Is individualization of sodium bicarbonate ingestion on time to peak necessary?
}

FARIAS DE OLIVEIRA, L.F., SAUNDERS, B., YAMAGUCHI, G., SWINTON, P. and GIANNINI ARTIOLI, G.

2020 


\section{Medicine \& Science in Sports \& Exercise \\ Is individualization of sodium bicarbonate ingestion based on time to peak necessary? --Manuscript Draft--}

\begin{tabular}{|c|c|c|}
\hline Manuscript Number: & \multicolumn{2}{|l|}{ MSSE-D-19-01075R1 } \\
\hline Article Type: & \multicolumn{2}{|l|}{ Original Investigation } \\
\hline Corresponding Author: & \multicolumn{2}{|l|}{$\begin{array}{l}\text { Guilherme Giannini Artioli } \\
\text { Sao Paulo, BRAZIL }\end{array}$} \\
\hline \multicolumn{3}{|c|}{ Corresponding Author's Institution: } \\
\hline \multicolumn{3}{|c|}{$\begin{array}{l}\text { Corresponding Author's Secondary } \\
\text { Institution: }\end{array}$} \\
\hline \multicolumn{3}{|c|}{ First Author Secondary Information: } \\
\hline \multirow[t]{5}{*}{ Order of Authors: } & \multicolumn{2}{|l|}{ Luana Farias de Oliveira } \\
\hline & \multicolumn{2}{|l|}{ Bryan Saunders } \\
\hline & \multicolumn{2}{|l|}{ Guilherme Yamaguchi } \\
\hline & \multicolumn{2}{|l|}{ Paul Swinton } \\
\hline & \multicolumn{2}{|l|}{ Guilherme Giannini Artioli } \\
\hline \multicolumn{3}{|c|}{ Order of Authors Secondary Information: } \\
\hline Funding Information: & $\begin{array}{l}\text { Fundação de Amparo à Pesquisa do } \\
\text { Estado de São Paulo } \\
(2016 / 50438-0)\end{array}$ & Dr Bryan Saunders \\
\hline
\end{tabular}


1 Article Title: Is individualization of sodium bicarbonate ingestion based on time to peak

2 necessary?

3 Submission type: original article

4 Running head: Repeated sodium bicarbonate supplementation

5

6 Authors: Luana Farias de Oliveira ${ }^{1}$, Bryan Saunders ${ }^{1,2}$, Guilherme Yamaguchi $^{1}$, Paul

$7 \quad$ Swinton $^{3}$, Guilherme Giannini Artioli ${ }^{1}$

8

9 Affiliations:

$10{ }^{1}$ Applied Physiology \& Nutrition Research Group; School of Physical Education and Sport;

11 Rheumatology Division; Faculdade de Medicina FMUSP, Universidade de Sao Paulo, Sao

12 Paulo, SP, BR.

$13{ }^{2}$ Institute of Orthopedics and Traumatology, Faculty of Medicine FMUSP, University of São

14 Paulo, Brazil.

$15{ }^{3}$ School of Health Sciences, Robert Gordon University, Aberdeen, United Kingdom

16

17 Corresponding author:

18 Guilherme Giannini Artioli

19 Applied Physiology \& Nutrition Research Group

20 University of São Paulo

21 Av. Mello de Moraes 65, Butantã

22 05508-030, Sao Paulo, SP, Brazil.

23 Phone: +55 11 3091-3096

24 Fax: +55 $113813-5921$

25 E-mail: $\underline{\text { artioli@usp.br }}$ 
ABSTRACT

Purpose: To describe thereliability of blood bicarbonate pharmacokinetics in response to sodium bicarbonate (SB) supplementation across multiple occasions and assess, using putative thresholds, whether individual variation indicated a need for individualised ingestion timings. Methods: Thirteen men (age $27 \pm 5$ y; body mass (BM) $77.4 \pm 10.5 \mathrm{~kg}$; height 1.75 $\pm 0.06 \mathrm{~m}$ ) ingested $0.3 \mathrm{~g} \cdot \mathrm{kg}^{-1} \mathrm{BM}$ SB in gelatine capsules on 3 occasions. One hour after a standardised meal, venous blood was obtained before and every 10 min following ingestion for $3 \mathrm{~h}$, then every $20 \mathrm{~min}$ for a further hour. Time-to-peak (Tmax), absolute-peak (Cmax), absolute-peak-change ( $\triangle \mathrm{Cmax})$ and area under the curve (AUC) were analysed using mixed models, intraclass correlation coefficient (ICC), coefficient of variation (CV) and typical error. Individual variation in pharmacokinetic responses was assessed using Bayesian simulation with multilevel models with random intercepts. Results: No significant differences between sessions were shown for blood bicarbonate regarding $\mathrm{Cmax}, \Delta \mathrm{Cmax}$ or AUC ( $p>0.05)$, although Tmax occurred earlier in SB2 (127 $\pm 36 \mathrm{~min})$ than in SB1 (169 \pm 54 min, $\mathrm{p}=0.0088)$ and SB3 $(159 \pm 42 \mathrm{~min}, \mathrm{p}=0.05)$. ICC, $\mathrm{CV}$ and typical error showed moderate to poor reliability. Bayesian modelling estimated that $>80 \%$ of individuals from the population experience elevated blood bicarbonate levels above $-6 \mathrm{mmol} \cdot \mathrm{L}^{-1}$ between $75-240$ min after ingestion, and between $90-225 \mathrm{~min}$ above $+6 \mathrm{mmol} \cdot \mathrm{L}^{-1}$. Conclusion: Assessing $\mathrm{SB}$ supplementation using discrete values showed only moderate reliability at the group level, and poor reliability at the individual level, while Tmax was not reproducible. However, when analysed as modelled curves, a $0.3 \mathrm{~g} \cdot \mathrm{kg}^{-1} \mathrm{BM}$ dose was shown to create a long-lasting window of ergogenic potential, challenging the notion that SB ingestion individualised to time-topeak is a necessary strategy, at least when SB is ingested in capsules.

Key words: time-course; ergogenic supplement; bioavailability; blood bicarbonate; reproducibility. 
INTRODUCTION

Sodium bicarbonate (SB) is an effective nutritional supplement to improve exercise performance and capacity during high intensity exercise (1-3). Acute ingestion of SB incurs an increase in blood $\mathrm{pH}$ and bicarbonate within approximately 30-60 mins which lasts up to several hours $(4,5)$. The metabolic alkalosis induced by SB ingestion leads to an increased efflux of lactate and hydrogen ions $\left(\mathrm{H}^{+}\right)$out of the working muscles during exercise (6), which can delay the negative impact of muscle acidosis on contractile processes (7) and improve exercise performance.

Despite the known ergogenic potential of SB supplementation, recent studies are moving away from typical mean group analyses towards individualised approaches $(8,9)$. This is due to the identification of factors that may moderate the ergogenic effect of SB, including variability in blood responses following SB ingestion. The time course of blood bicarbonate responses to acute SB ingestion indicates large variability between individuals, with peak bicarbonate concentration occurring between 75 and 180 minutes when ingested in capsules (4) and between 10 and 140 minutes $(\underline{5}, \underline{10})$ in solution using the commonly employed relative dose of $0.3 \mathrm{~g} \cdot \mathrm{kg}^{-1}$ body mass (BM) of SB. Coupled with recent evidence demonstrating consistent intraindividual response to the same dose taken on different days, it has been suggested that the optimal time to perform exercise would be at this time at which blood bicarbonate peaks ( 8 ). However, only one study to date has investigated the reproducibility of these blood response across two sessions providing SB in solution (5). In addition, the timecourse responses to SB ingestion when meal ingestion is controlled remain unknown, a procedure that is likely used by most athletes in real competitive situations. Thus, more information about the consistency of the time-course responses to SB ingestion is warranted, particularly after the ingestion of a standardised meal. 
The ergogenic effects of SB have been suggested to be dependent on a minimum increase of circulating bicarbonate, with an increase of $+5 \mathrm{mmol} \cdot \mathrm{L}^{-1}$ being considered a zone of potential ergogenic benefit, and increases above $6 \mathrm{mmol} \cdot \mathrm{L}^{-1}$ being almost certainly ergogenic $(\underline{4}$, 11, 12). It is currently unclear whether the absolute increases at time to peak differ substantially from those generally seen at standardised time points. The mean $+6.5 \pm 1.3$ $\mathrm{mmol} \cdot \mathrm{L}^{-1}$ increase shown at time to peak by Gough, et al. (13) is similar to the increases shown following 60 min (+6.1 Dias, et al. (14); +5.1 Jones, et al. (4); +5.7 Gough, et al. (8)), $90 \mathrm{~min}$ (+6.5 Jones, et al. (4) +6.1 Gough, et al. (8) and 120 min (+6.5 Jones, et al. (4); +5.6 Gough, et al. (8)) with the same $0.3 \mathrm{~g} \cdot \mathrm{kg}^{-1} \mathrm{BM}$ dose. Furthermore, blood bicarbonate concentration was not shown to be different 60-, 120- and 180-min following SB supplementation in gelatine capsules (15), which raises questions as to whether ingestion timing is an important factor for the ergogenic effects of SB in this form. It remains to be determined whether blood bicarbonate is consistently increased close to peak, or above $+6 \mathrm{mmol} \cdot \mathrm{L}^{-1}$, for prolonged periods.

Although time to peak in blood bicarbonate has been touted as a strategy to optimise SB ingestion (13), there are several limitations that may preclude its applicability to actual training or competition settings. Firstly, it requires athletes or coaches to have access to a reliable blood gas analyser and to perform a subsequent time-course measurement of blood bicarbonate responses to SB ingestion over several hours. This procedure is laborious, costly and not easily accessible for most athletes. Secondly, time to peak assumes that the increases in circulating SB are substantially greater when blood bicarbonate peaks than at standard time points, instead of assuming that blood bicarbonate will fluctuate around the peak value for a period of time. An in-depth analysis of the blood bicarbonate responses to SB ingestion could reveal whether the "window of ergogenicity" is limited to a fixed time point or extends across a broad time period following SB ingestion. This could provide important practical information for athletes as to whether determination of time-to-peak is a necessary strategy. 

determine the reliability of orally ingested SB pharmacokinetics over 4 hours using multiple testing occasions (including a placebo trial). A secondary aim of this study was to assess whether individual variation in orally ingested SB pharmacokinetics indicated a need for individualised ingestion timings. Our hypothesis was that SB ingestion would result in a sustained increase in blood bicarbonate above the purported ergogenic thresholds. We also hypothesised that this pattern would result in inconsistent responses in Tmax, potentially challenging the need for individualised ingestion timings.

\section{METHODS}

Participants

Twenty-four young, physically active, healthy men were screened for eligibility; three of them did not meet inclusion criteria, and six other candidates did not wish to partake in the study. Fifteen participants enrolled in the study, but one withdrew after the first session due to personal reasons while a second participant withdrew after the third session due to gastric distress associated with SB ingestion. Therefore, complete data were obtained for 13 participants and used in all analyses herein reported (age $=27 \pm 5$ years; $B M=77.4 \pm 10.5 \mathrm{~kg}$; height $=1.75 \pm 0.06 \mathrm{~m}$; body mass index $=25.2 \pm 2.9 \mathrm{~kg} \cdot \mathrm{m}^{2}$ ). Inclusion criteria were defined a priori as: healthy men aged 18 to 35 years. Exclusion criteria were defined a priori as: smoking, use of medications that may alter stomach $\mathrm{pH}$ and any diagnosed condition that could affect the gastrointestinal and blood $\mathrm{pH}$ balance. All volunteers were informed about the discomforts and risks associated with participation and thereafter provided written consent. The study was approved by the Institutional Ethics Committee (29181114.0.0000.5391). 


\section{Study Design}

This was a crossover, placebo-controlled study in which volunteers visited the laboratory on four separate occasions, 2-7 days apart, to receive SB (on 3 occasions) or placebo (PL, on 1 occasion). To control for order effects, treatments were randomly assigned to each visit in a balanced fashion using the Latin square. Participants were requested to refrain from strenuous physical activity and alcohol intake in the $24 \mathrm{~h}$ preceding each visit. They were also instructed to maintain a similar pattern of food intake on all days prior to the tests. Compliance with these requests was verbally confirmed with all participants. The participants arrived at the laboratory in the morning after an overnight fast, and a standardised breakfast (energy: 563 kcal; protein: $9.3 \mathrm{~g}$; carbohydrate: $89.6 \mathrm{~g}$; fats: $8.9 \mathrm{~g}$ ) was served to avoid variations in blood responses due to differences in food intake prior to the tests. One hour following the breakfast, blood samples were taken before and during 4 hours after the ingestion of SB or PL.

\section{Supplementation protocol}

Sodium bicarbonate $\left(0.3 \mathrm{~g} \cdot \mathrm{kg}^{-1} \mathrm{BM}\right.$; Farmácia Analítica, Rio de Janeiro, Brazil) was given on three different visits while an identical number of capsules was provided in PL (each capsule containing 56 mg of corn flour; Farmácia Analítica, Rio de Janeiro, Brazil).

Supplements were given in gelatine capsules identical in size and appearance. Participants had 5 minutes to ingest all capsules. After ingestion of the last capsule, a stopwatch was started to control the exact times at which blood samples were to be taken.

\section{Blood sampling}

The cephalic vein was cannulated (catheter 20 G Safelet Nipro) and kept warm with the use of a forearm thermal blanket maintained at $48^{\circ} \mathrm{C}$ throughout the entire $4 \mathrm{~h}$ sampling period. A venous blood sample was taken for the determination of baseline blood parameters (i.e., before ingestion). The participants then ingested SB or PL in gelatine capsules along with 400 $\mathrm{ml}$ of water and then $100 \mathrm{ml}$ per hour throughout. Following ingestion, blood samples were 
taken every 10 minutes for 3 hours, and then every 20 minutes in the $4^{\text {th }}$ hour. Blood samples

$(1 \mathrm{ml})$ were collected in heparinised syringes and immediately analysed for $\mathrm{pH}$ and pCO2 using a blood gas analyser (RAPIDLab 348, Siemens, Germany). Quality controls were performed each experimental day prior to data collection. Blood bicarbonate was calculated using the Henderson-Hasselbalch equation. The inter-assay coefficient of variation of blood bicarbonate was $6.4 \%$ (determined over the 4-h period during the PL trial). Blood bicarbonate was defined $a$ priori as the primary outcome.

Side-effects

Side-effects were recorded at the same time-points as blood collection using an adapted models were also used to compare blood bicarbonate concentration at Tmax and 60, 90 and 120 minutes after ingestion. To account for the time series nature of the data and subsequent 
173 Autoregressive, Toeplitz and Unstructured) were tested to verify the model that best fit to each 174 data set, according to the Bayesian Information Criterion (lowest BIC value). Pairwise comparisons adjusted by Tukey-Kraemer were used when a significant F-value was observed. Intraclass correlation coefficient (ICC), typical error using data from the $3 \mathrm{SB}$ trials to determine within-subject reliability. Test-retest coefficient of variations (CV) were calculated using the mean square root method (17). The frequency of side-effects reported between visits, irrespective of intensity and duration, was analysed using the chi-square test. Side-effect scores for the 13 symptoms were summed within each visit and compared between visits using the Friedman Test. Statistical significance was accepted at $p \leq 0.05$. Inter-trial reliability was assessed by calculating typical errors (sigma) and ICCs from level 0 and level 1 residuals in the mixed models. Since all blood $\mathrm{pH}$ data and analysis were similar that of blood bicarbonate, herein we report blood bicarbonate data only although blood $\mathrm{pH}$ data is included as supplemental material (Supplemental Digital Content 1 - Figure - Blood pH responses).

To describe individual variation in the pharmacokinetic responses to orally ingested SB and assess the need for individualised ingestion timings, a Bayesian perspective was adopted. A Bayesian perspective best facilitated probabilistic questions such as the probability of an individual's blood bicarbonate level exceeding a given absolute increase (i.e. +5 or $+6 \mathrm{mmol} \cdot \mathrm{L}^{-}$

$\left.{ }^{1}\right)$ or percentage increase within specific time windows. Using data collected across the participants' three active testing sessions, Bayesian multilevel models with random intercepts and slopes were fitted using the brms package $(\underline{18})$ in the programming language $\mathrm{R}$. In contrast to treating observed data as independent points, it was assumed that changes in blood bicarbonate after SB ingestion followed a smooth response that could be adequately described by a polynomial function. Linear, quadratic, cubic and quartic models were fitted, with Watanabe-Akaike Information Criterion (WAIC) used to identify a cubic model as the best fit for further evaluation. The Bayesian analysis required specification of prior beliefs regarding 
model parameters. To reflect a lack of prior information, default improper flat priors were selected for population-level regression parameters and the LKJ-prior selected for the multivariate normal distribution covariance matrix between group-level parameters. Posterior estimates of size $n=10,000$ were generated for each parameter using MCMC sampling with 4 chains and 3,500 iterations (warmup $=1,000$ iterations). These posterior estimates described the typical (e.g. median) blood bicarbonate response representative of the group. To explore the likely range and distribution of responses across individuals from a similar population, posterior estimates were used to probabilistically sample regression parameters from a multivariate normal distribution. For each parameter set $(n=10,000), 100$ individual blood bicarbonate traces (each a cubic polynomial) were produced and the total pool of 1 million traces used to estimate probabilities that an individual's blood bicarbonate increased above +5 and $+6 \mathrm{mmol} \cdot \mathrm{L}^{-1}$. A threshold of $80 \%$ probability was selected to assist with interpretation of results and identify time windows where for practical purposes it could be concluded that the vast majority of individuals met the criteria.

\section{RESULTS}

Reliability

Blood bicarbonate at baseline was not different between sessions $(\mathrm{SB} 1=25.7 \pm 2.4$; $\mathrm{SB} 2=25.0 \pm 2.0 ; \mathrm{SB} 3=26.0 \pm 1.7 ; \mathrm{PL}=25.4 \pm 2.1 \mathrm{mmol} \cdot \mathrm{L}^{-1} ; \mathrm{F}=0.74 ; p=0.5348 ;$ Figure 1). Reliability statistics were calculated for baseline (TE: 1.7 units, ICC: 0.26), Cmax (TE: 2.0 units, ICC: 0.20), DeltaCmax (TE: 2.5 units, ICC: 0.1) and Tmax (TE: 38.7 units, ICC: 0.34). ICCs, typical error and CVs calculated for blood bicarbonate between sessions are presented in Table 1.

Area under the curve was not different between SB sessions $(\mathrm{SB} 1=1447 \pm 364$ $\mathrm{mmol} \cdot \min \cdot \mathrm{L}^{-1} ; \mathrm{SB} 2=1468 \pm 421 \mathrm{mmol} \cdot \mathrm{min} \cdot \mathrm{L}^{-1} ; \mathrm{SB} 3=1210 \pm 520 \mathrm{mmol} \cdot \mathrm{min} \cdot \mathrm{L}^{-1} ; \mathrm{F}=0.87$ 
$p=0.43$; figure 1, panel B). No significant differences between sessions were shown for blood bicarbonate regarding Cmax $\left(\mathrm{SB} 1=36.8 \pm 2.8 \mathrm{mmol} \cdot \mathrm{L}^{-1} ; \mathrm{SB} 2=35.5 \pm 1.4 \mathrm{mmol} \cdot \mathrm{L}^{-1} ; \mathrm{SB} 3=\right.$ $35.2 \pm 2.0 \mathrm{mmol} \cdot \mathrm{L}^{-1} ; \mathrm{F}=2.65 ; p=0.10 ;$ figure 1 , panel $\left.\mathrm{C}\right)$ or $\Delta \mathrm{Cmax}\left(\mathrm{SB} 1=11.1 \pm 2.7 \mathrm{mmol} \cdot \mathrm{L}^{-}\right.$ ${ }^{1} ; \mathrm{SB} 2=10.5 \pm 2.5 \mathrm{mmol} \cdot \mathrm{L}^{-1} ; \mathrm{SB} 3=9.3 \pm 2.2 \mathrm{mmol} \cdot \mathrm{L}^{-1} ; \mathrm{F}=1.30 ; p=0.29$, figure 1, panel D), although Tmax occurred significantly earlier in SB2 (127 $\pm 36 \mathrm{~min})$ than in SB1 $(169 \pm 54$ min, $p=0.0088)$ and SB3 (159 $\pm 42 \min , p=0.05$; Figure 2) (main effect of session: $\mathrm{F}=5.83$; $p=0.0086$ ) (figure 1, panel E).

Individual analysis showed substantial intra-individual variation for Tmax in blood bicarbonate following SB ingestion, despite the lack of statistical differences between sessions for mean values (figure 2). Moreover, a prolonged time period above the $+5 \mathrm{mmol} \cdot \mathrm{L}^{-1}$ (light grey blocks) and $+6 \mathrm{mmol} \cdot \mathrm{L}^{-1}$ (dark grey blocks) thresholds was shown in nearly all participants in all three sessions (figure 2).

\section{Tmax vs. standard time points}

Comparison between Tmax and standard time points showed statistically significant differences in absolute bicarbonate values between all prespecified time points (Tmax: $35.9 \pm$ $2.2 \mathrm{mmol} \cdot \mathrm{L}^{-1} ; 60 \mathrm{~min}: 30.8 \pm 2.4 \mathrm{mmol} \cdot \mathrm{L}^{-1} ; 90 \mathrm{~min}: 32.1 \pm 2.6 \mathrm{mmol} \cdot \mathrm{L}^{-1} ; 120 \mathrm{~min} 33.0 \pm 3.0$ $\left.\mathrm{mmol} \cdot \mathrm{L}^{-1} ; \mathrm{F}=45.87 ; p<0.0001\right)$, except for $90 v s 120 \mathrm{~min}(p=0.1852)$. Delta change for blood bicarbonate was different between Tmax vs. all pre specified time points (all $p<0.001$ ), but no significant differences were shown between 90 and $120 \mathrm{~min}(\mathrm{p}=0,1852$; Figure 3 ).

\section{Modelling approaches}

Bayesian modelling and subsequent simulations estimate that over $80 \%$ of individuals from the population experience elevated blood bicarbonate levels greater than $5 \mathrm{mmol} \cdot \mathrm{L}^{-1}$ between 75 and $240 \mathrm{~min}$ after ingestion. For absolute increases greater than $6 \mathrm{mmol} \cdot \mathrm{L}^{-1}$, the expected window decreased to between 90 and 225 min (Table 2). Results of the Bayesian 
modelling and subsequent simulations with a multilevel cubic model are illustrated in Figure

248

249

250

251

252

253

254

255

256

257

258

259

260

261

262

263

264

265

266

267

268

269

4.

\section{Side-effects}

All participants reported one or more side-effects in each of the three SB trials, with a total of 39 symptoms being reported in SB1, 46 symptoms in SB2 and 37 symptoms in SB3. No significant differences between sessions were shown for the frequency of side-effects symptoms $\left(\mathrm{x}^{2}=1.45, p<0.485\right)$. The Friedman test showed that intensity of symptoms throughout the time-course was not different between visits ( $p=0.7627$; Supplemental Digital Content 2 - Figure - Side-effects).

\section{DISCUSSION}

This study is the first to investigate a 4-h time-course response of blood bicarbonate, $\mathrm{pH}$ and side-effects following the ingestion of $0.3 \mathrm{~g} \cdot \mathrm{kg}^{-1} \mathrm{BM}$ sodium bicarbonate in gelatine capsules on 3 distinct sessions. We hypothesised that, due to the dynamic nature of blood acidbase regulation and natural fluctuation in blood bicarbonate concentration, a single time point for peak blood bicarbonate would not properly represent the sustained increase in blood bicarbonate following acute SB ingestion Jones, et al. (4). We also sought to gather further information on the within-subject consistency of blood bicarbonate responses to acute SB ingestion in gelatine capsules. Repeated administration of SB in gelatine capsules did not elicit consistent responses for bicarbonate Tmax, which is in agreement with our initial hypothesis, and potentially challenges the necessity of individualised ingestion timings. Overall, our results indicate that blood bicarbonate continuously rises for $\sim 120-160$ min after SB ingestion before reaching a plateau, with elevated values being shown until the end of the 4-h period.

The Bayesian analysis revealed an interesting pattern of elevated probabilities of increased blood bicarbonate levels (above the theoretical ergogenic threshold) from $\sim 60 \mathrm{~min}$ 
after ingestion to the end of the measurement period. Although performance assessment was beyond the objectives of this study, our data might challenge the notion that a single time point at which blood bicarbonate peaks is necessary to optimise the ergogenic effects of SB. Instead, the Bayesian model and reliability analyses, collectively, suggest that it is not possible to accurately determine when peak blood bicarbonate has been reached since slight variations in blood bicarbonate, including the peak values, are most likely due to random error (owing to measurement error and biological variation) around the already elevated blood bicarbonate concentrations. Therefore, it appears that the ergogenic potential of SB is likely to be in place for at least 3 hours, starting $\sim 60$ min after ingestion. This finding is consistent with a previous study that measured blood bicarbonate for 3 hours in response to SB ingestion and found a similar plateau-shaped curve of increased blood bicarbonate_(4). However, our data contrasts with another similar study that showed a trend towards a rapid decline in blood bicarbonate after reaching its peak (8). Perhaps the best explanation for the difference between these studies may be related to the form of SB administration. While our study and Jones, et al. (4) provided SB in gelatine capsules and found a more sustained increase in blood bicarbonate, Gough, et $\underline{\text { al. }}$ (8) provided SB in solution and found a more rapid profile of blood bicarbonate appearance and disappearance. These differences in the shape of the blood bicarbonate curves (i.e., more sustained $v s$. rapid decline) seems to also explain why the reliability of Tmax was poor in our study (random error around a long-lasting elevation in blood bicarbonate) in contrast with a good reliability in the study by Gough et al. (sharp peak and rapid decline allow a clear identification of Tmax). There is a slight difference in pharmacokinetics when SB is ingested in capsules compared to SB ingested in solution (19), meaning any conclusions in this paper are restricted to supplementation in gelatine capsules.

Another important difference between studies is the provision of a meal before SB ingestion. While we started blood collection one hour following a standardised breakfast, 
Gough, et al. (8) requested their participants to refrain from food 4 hours before SB ingestion. It is possible that the time at which an individual consumes their pre-competition or training meal influences the subsequent response to SB ingestion. Although unexplored, the influence of meal ingestion on the pharmacokinetic responses to SB is of great practical implication. In our study, we opted to provide a standardised breakfast to better simulate a practical training or competition situation, assuming that athletes typically train or compete in a well-fed postprandial state. It must be noted, however, that although our pre-ingestion meal strategy represents the responses to SB ingestion under a general post-prandial state, we did not explore the impact of meal composition on these responses, which remain a largely overlooked topic of investigation. Another interesting point is that our $\Delta \mathrm{Cmax}$ values $\left(\sim+10 \mathrm{mmol} \cdot \mathrm{L}^{-1}\right)$ were considerably higher than the $+7 \mathrm{mmol} \cdot \mathrm{L}^{-1}$ shown by Gough, et al. (8) when supplemented with the same $0.3 \mathrm{~g} \cdot \mathrm{kg}^{-1} \mathrm{BM}$ dose of $\mathrm{SB}$. We speculate that this too could be explained, at least in part, by the timing of food intake prior to supplementation. Since our volunteers had eaten only one hour before supplementation, they could have been presenting a slight metabolic alkalosis due to the "alkaline tide" effect that accompanies food ingestion (20). Alternatively, the presence of food in their stomach could have resulted in higher luminal $\mathrm{pH}(21)$, which could result in less bicarbonate reacting with stomach acids, allowing more bicarbonate to enter the intestine to be absorbed. Differences in blood gas analysers and in blood collection methods (e.g., vein vs. capillary blood taken with or without arterialisation) may have also played some role in the different results between studies; however, it is important to note that different methods may yield different absolute values but they unlikely will result in an entirely different pharmacokinetic curve.

Analysis of classical timings of bicarbonate supplementation (60, 90 and 120 min post ingestion) identified a progressive step pattern with significant increases over each $30 \mathrm{~min}$ period. On average, blood bicarbonate at time to peak was $2.4 \mathrm{mmol} \cdot \mathrm{L}^{-1}$ higher than that 
obtained 120 minutes post ingestion. However, given typical error at baseline was estimated as $1.8 \mathrm{mmol} \cdot \mathrm{L}^{-1}$, differences can be explained by random errors, especially given the large number of data points measured and the probable extended plateau period. Nevertheless, mean values were very near or above the purported ergogenic thresholds in all time points. Importantly, there is currently no evidence for a linear association between the magnitude of the blood bicarbonate increase with the magnitude of the ergogenic effect of SB. Thus, one cannot assume that the higher the blood bicarbonate value, the greater the effects on performance. In fact, evidence so far points towards a minimum increase in blood bicarbonate necessary for SB to exert its ergogenic effects $(\underline{4}, \underline{11}, 12)$. In that sense, the Bayesian modelling presents a significant advance in data interpretation, as it allows for direct probabilistic questions to be addressed. For example, models can be used to estimate the probability that an individual from the population will experience an increase of at least $+5 \mathrm{mmol} \cdot \mathrm{L}^{-1}$ (or any other value) over a specified time interval. The Bayesian modelling clearly indicated a high probability for ergogenic effects (assuming the validity of the +5 and $+6 \mathrm{mmol} \cdot \mathrm{L}^{-1}$ thresholds) over a prolonged period of time although there was large inter-individual variability (Figure 2). Future research should corroborate the use of these ergogenic thresholds for exercise performance.

Another aim of our study was to confirm whether blood bicarbonate responses and, more importantly, the time to peak in blood bicarbonate, are consistent across 3 identical trials conducted on different days. Although Cmax and $\Delta \mathrm{Cmax}$ were similar between trials, we showed a significant difference in Tmax between trials, indicating poor repeatability of this measure. ICC and CV also showed moderate-to-poor reliability for these variables, especially $\Delta \mathrm{Cmax}$ and Tmax. In support of this, individual analysis also showed a considerable intraindividual variability in blood bicarbonate responses to acute SB ingestion (Figure 2). Thus, we suggest that determination of Tmax for subsequent implementation prior to exercise may not be the most suitable method when ingesting SB in gelatine capsules. This moderate-to-poor 
reliability for blood bicarbonate measures shown in our study is somewhat in contrast with recent studies that showed consistent blood bicarbonate responses between trials $(\underline{8}, \underline{14})$, but in agreement with a study that showed larger intra-individual variation in blood responses to SB ingestion (22). The large variation shown here may be a reflection of the long plateau-shaped curve we showed for blood bicarbonate, where values fluctuate around Cmax for a prolonged period, allowing the peak value to occur anytime within this period of time. This reinforces the notion that the peak value is, in our case, only slightly different than the other similarly elevated values, and that identification of a solitary peak value might represent random variation rather than a true peak value which would coincide with the best opportunity for SB to be ergogenic. Therefore, it appears likely that there is a broad window of opportunity, and not a single time point, where SB supplementation is more likely to be effective. This is supported by the Bayesian modelling used in the current study, and by previous studies showing no differences in the performance effects of SB between different time points following ingestion (23) $)$. Again, the differences between our results and those by Gough, et al. (8) might be due to different occurrence of important side-effects remains a major obstacle for SB use in practical settings, and this is yet to be solved. Future studies should look for ways to promote the ergogenic effects 

the highest possible external validity, we acknowledge that the participants remained rested for the entire experimental protocol. This means that the commencement of exercise, either a warm-up or a competition, could alter the time-responses shown herein. The exact window of ergogenic potential shown here can only be assumed to be valid if the athlete remains rested between SB ingestion and the beginning of the exercise. Future studies should examine how exercise of different intensities affect the pharmacokinetics and the time course of ergogenic properties of SB. Another limitation is the use of a single $0.3 \mathrm{~g} \cdot \mathrm{kg}^{-1} \mathrm{SB}$ dose, which does not allow any extrapolation of the current findings to smaller doses (e.g., $0.2 \mathrm{~g} \cdot \mathrm{kg}^{-1}$ ) or other supplementation strategies (e.g., split-dose strategy). In fact, because previous studies showed a shorter period of blood bicarbonate elevations (above the purported ergogenic thresholds) with smaller doses (4), it is possible that time to peak remains as a relevant strategy when smaller doses are used, although this is yet to be confirmed. Indeed, the study by Gough, et al. (13) showed that individualised strategies based on time-to-peak may allow for the use of smaller doses without any measurable loss in SB ergogenicity. However, this study did not directly compare the effect of SB at time to peak with standard time points that are typically used in SB literature (e.g. 60-, 90- or 120-min following SB ingestion). Thirdly, we were unable to perform $\mathrm{PO}_{2}$ analysis in our samples, meaning we could not ensure venous blood arterialisation, despite the use of a thermal blanket specifically designed for the arterialisation of venous blood in the forearm. Lastly, the interpretation of our data is based on the current assumption that increases in +5 and $+6 \mathrm{mmol} \cdot \mathrm{L}^{-1}$ in blood bicarbonate are true thresholds for SB to be ergogenic. Since we were unable to associate the pharmacokinetic data with true performance effects in our participants, some caution should be exercised when extrapolating our findings to performance. 

breakfast across three sessions showed only moderate reliability at the group level, but at the individual level, reliability appears to be poor. In particular, Tmax was not reproducible across the three sessions, suggesting it may not the most effective way by which to optimise SB supplementation. This is probably related to the long, sustained increases in blood bicarbonate following SB ingestion, so that solitary peak values are more a reflection of random error rather than true maximal increases in blood bicarbonate. Nonetheless, our data show that a $0.3 \mathrm{~g} \cdot \mathrm{kg}^{-} 1$

$\mathrm{BM}$ dose results in a long-lasting ( $\sim 3$ hours, staring from $\sim 60$ min after SB ingestion) window of ergogenic potential considering an ergogenic threshold of $+5-6 \mathrm{mmol} \cdot \mathrm{L}^{-1}$ in blood bicarbonate from baseline. This challenges the notion that SB ingestion individualised to time to peak is a necessary strategy, at least when a dose of $0.3 \mathrm{~g} \cdot \mathrm{kg}^{-1}$ is taken in gelatine capsules.

406

407

\section{ACKNOWLEDGMENTS}

The authors would like to extend their gratitude to the volunteers who participated in this study, Rodrigo Abdalla and Farmácia Analítica (Rio de Janeiro, Brazil) for providing the sodium bicarbonate and placebo supplements at no cost for this study.

\section{FUNDING AND CONFLICT OF INTEREST}

This study was financed in part by the Coordenação de Aperfeiçoamento de Pessoal de Nível

414 Superior - Brasil (CAPES) - Finance Code 001. Bryan Saunders (2016/50438-0 and 415 2017/04973-4) has been financially supported by Fundação de Amparo à Pesquisa do Estado 416 de São Paulo.

417 The authors declare no conflict of interest. No financial support was received. The results of 418 this study are presented clearly, honestly, and without fabrication, falsification, or inappropriate 
data manipulation. The results of present study do not constitute endorsement by the American College of Sports Medicine.

421

422

\section{REFERENCES}

423 2016;15(4):233-44.

427

2. Carr AJ, Hopkins WG, Gore CJ. Effects of acute alkalosis and acidosis on performance: a meta-analysis. Sports Med. 2011;41(10):801-14.

3. Peart DJ, Siegler JC, Vince RV. Practical recommendations for coaches and athletes: a meta-analysis of sodium bicarbonate use for athletic performance. J Strength Cond Res. 2012;26(7):1975-83.

4. Jones RL, Stellingwerff T, Artioli GG, Saunders B, Cooper S, Sale C. Dose-Response of Sodium Bicarbonate Ingestion Highlights Individuality in Time Course of Blood Analyte Responses. Int J Sport Nutr Exerc Metab. 2016;26(5):445-53.

5. Gough LA, Deb SK, Sparks AS, McNaughton LR. The Reproducibility of Blood Acid Base Responses in Male Collegiate Athletes Following Individualised Doses of Sodium Bicarbonate: A Randomised Controlled Crossover Study. Sports Med. 2017;47(10):2117-27.

6. Mainwood GW, Worsley-Brown P. The effects of extracellular $\mathrm{pH}$ and buffer concentration on the efflux of lactate from frog sartorius muscle. The Journal of physiology. 1975;250(1):1-22. 
442 7. Woodward M, Debold EP. Acidosis and Phosphate Directly Reduce Myosin's ForceGenerating Capacity Through Distinct Molecular Mechanisms. Front Physiol. 2018;9:862.

8. Gough LA, Deb SK, Sparks AS, McNaughton LR. The Reproducibility of Blood Acid Base Responses in Male Collegiate Athletes Following Individualised Doses of Sodium Bicarbonate: A Randomised Controlled Crossover Study. Sports medicine. 2017.

9. Gough LA, Deb SK, Brown D, Sparks SA, McNaughton LR. The effects of sodium

14. Dias GFdA, Silva VdE, Painelli VdS et al. (In)Consistencies in Responses to Sodium and Double-Blind Study. PloS one. 2015;10(11):e0143086. 
15. Siegler JC, Marshall PW, Bray J, Towlson C. Sodium bicarbonate supplementation and ingestion timing: does it matter? J Strength Cond Res. 2012;26(7):1953-8.

16. Jeukendrup AE, Vet-Joop K, Sturk A et al. Relationship between gastro-intestinal complaints and endotoxaemia, cytokine release and the acute-phase reaction during and after a long-distance triathlon in highly trained men. Clinical science. 2000;98(1):4755.

17. Hyslop NP, White WH. Estimating precision using duplicate measurements. Journal of the Air \& Waste Management Association. 2009;59(9):1032-9.

18. Bürkner P-C. brms: An R package for Bayesian multilevel models using Stan. Journal of Statistical Software. 2017;80(1):1-28.

19. Hilton NP, Leach NK, Sparks SA et al. A Novel Ingestion Strategy for Sodium Bicarbonate Supplementation in a Delayed-Release Form: a Randomised Crossover Study in Trained Males. Sports medicine - open. 2019;5(1):4.

20. Niv Y, Fraser GM. The alkaline tide phenomenon. Journal of clinical gastroenterology. 2002;35(1):5-8.

21. Dressman JB, Berardi RR, Dermentzoglou LC et al. Upper gastrointestinal (GI) pH in young, healthy men and women. Pharmaceutical research. 1990;7(7):756-61.

22. Carr AJ, Slater GJ, Gore CJ, Dawson B, Burke LM. Reliability and effect of sodium bicarbonate: Buffering and 2000-m rowing performance. International journal of sports physiology and performance. 2012;7(2):152-60.

23. Siegler JC, Marshall PWM, Bray J, Towlson C. Sodium Bicarbonate Supplementation and Ingestion Timing: Does It Matter? J Strength Cond Res. 2012;26(7):1953-8. 
Figure 1. Panel A: Time course of mean blood bicarbonate $\left(\mathrm{HCO}_{3}^{-}\right)$responses following

491

492

493

494

495

496

497

498

499

500

501

502

503

504

505

506

507

508

509

510

511

512 Figure 4. Density plot of simulated $(\mathrm{n}=1$ million) cubic time-course of blood bicarbonate supplementation, determined during each of the 3 sodium bicarbonate (SB1 black circle; SB2 dark grey square; SB3 light grey triangle) and placebo (PL; stars) trials, * different from PL; Panel B: Area under the curve (AUC) for blood bicarbonate in the 3 SB sessions; Panel C: Peak blood bicarbonate concentration (Cmax) in the 3 SB sessions; Panel D: maximum increase in blood bicarbonate from baseline $(\Delta \mathrm{Cmax})$ in the $3 \mathrm{SB}$ sessions; Panel E: Time to peak (Tmax), determined in the $3 \mathrm{SB}$ sessions. Individual data are presented in circles; bars and error bars represent group mean and standard deviation, $p$-values represent adjusted within-subject effects.

Figure 2. Individual data for blood bicarbonate increases after sodium bicarbonate supplementation $(\mathrm{SB})$, on the three visits. Black bricks = peak bicarbonate concentration (Tmax); bricks filled with diagonal lines $=+6 \mathrm{mmol} \cdot \mathrm{L}^{-1}$ or above; grey bricks $=+5-+5.9$ $\mathrm{mmol} \cdot \mathrm{L}^{-1}$

Figure 3. Maximum increase in blood bicarbonate $\left(\mathrm{HCO}_{3}{ }^{-}\right)$from baseline at classical timing points following sodium bicarbonate supplementation (60, 90 and 120 minutes following ingestion) and at time-to-peak (Tmax; $152 \pm 47$ minutes) determined from the $3 \mathrm{SB}$ sessions. Dotted line at 5 and $6 \mathrm{mmol} \cdot \mathrm{L}^{-1}$ represents the theoretical thresholds of potential and almost certain ergogenic effects. Bars and error bars represent means and standard deviation across the three SB sessions. $p$-values represent adjusted within-subject effects.

513 across a 4-h period following the acute ingestion of $0.3 \mathrm{mg} \cdot \mathrm{kg}^{-1}$ body mass of sodium 
514 bicarbonate. White triangles represent mean values from the data, and darker areas represent

515 blood bicarbonate values with greater probabilities to occur.

516

517 Supplemental figure 1: Panel A: Time course of mean $\mathrm{pH}$ responses following 518 supplementation, determined during each of the 3 sodium bicarbonate (SB1 black circle; SB2 519 dark grey square; SB3 light grey triangle) and placebo (PL; stars) trials, * different from PL; 520 Panel B: Area under the curve (AUC) for $\mathrm{pH}$ in the 3 SB sessions; Panel C: $\mathrm{pH}$ peak (Cmax) 521 in the $3 \mathrm{SB}$ sessions; Panel D: maximum increase in $\mathrm{pH}$ from baseline $(\Delta \mathrm{Cmax})$ in the $3 \mathrm{SB}$ 522 sessions; Panel E: Time to peak (Tmax) for $\mathrm{pH}$, determined in the $3 \mathrm{SB}$ sessions. Individual 523 data are presented in circles; bars and error bars represent group mean and standard deviation.

525 Supplemental figure 2: Side-effects. Size of circle refers the maximal side-effects intensity 526 related, where larger means more intense. 
Table $1 .{ }^{1}$

\begin{tabular}{|c|c|c|c|c|c|c|c|c|c|}
\hline \multirow{3}{*}{$\begin{array}{l}\text { Time points } \\
(\text { min) }\end{array}$} & \multicolumn{3}{|c|}{ Intraclass correlation } & \multicolumn{3}{|c|}{$\begin{array}{l}\text { Typical error } \\
\left(\mathbf{m m o l} \cdot \mathbf{L}^{-1}\right)\end{array}$} & \multicolumn{3}{|c|}{$\begin{array}{c}\text { Coefficient of Variation } \\
(\%)\end{array}$} \\
\hline & & \multicolumn{2}{|c|}{ confidence interval } & & \multicolumn{2}{|c|}{ confidence interval } & SB1 vs & SB2 vs & SB1 vs \\
\hline & & 2.5 & 97.5 & & 2.5 & 97.5 & SB2 & SB3 & SB3 \\
\hline Baseline & 0.389 & 0.208 & 0.665 & 1.77 & 1.64 & 1.94 & 5.88 & 6.97 & 7.65 \\
\hline 10 & 0.330 & 0.080 & 0.681 & 1.44 & 1.10 & 1.96 & 3.31 & 5.72 & 6.38 \\
\hline 20 & 0.268 & 0.002 & 0.664 & 1.92 & 1.44 & 2.62 & 9.40 & 8.25 & 8.63 \\
\hline 30 & 0.218 & 0.002 & 0.611 & 2.09 & 1.60 & 2.79 & 7.63 & 6.16 & 8.66 \\
\hline 40 & 0.453 & 0.037 & 0.764 & 1.93 & 1.47 & 2.71 & 6.20 & 4.92 & 8.87 \\
\hline 50 & 0.335 & 0.010 & 0.698 & 2.11 & 1.63 & 2.85 & 6.28 & 5.40 & 8.75 \\
\hline 60 & 0.318 & 0.007 & 0.709 & 2.16 & 1.66 & 2.92 & 7.34 & 6.03 & 6.24 \\
\hline 70 & 0.367 & 0.016 & 0.726 & 1.70 & 1.29 & 2.33 & 4.90 & 4.76 & 6.38 \\
\hline 80 & 0.361 & 0.013 & 0.717 & 1.80 & 1.37 & 2.50 & 4.03 & 6.86 & 5.04 \\
\hline 90 & 0.388 & 0.042 & 0.740 & 2.26 & 1.73 & 3.10 & 5.91 & 5.05 & 8.55 \\
\hline 100 & 0.338 & 0.007 & 0.686 & 2.43 & 1.88 & 3.33 & 6.64 & 7.10 & 7.94 \\
\hline 110 & 0.263 & 0.003 & 0.645 & 2.81 & 2.16 & 3.79 & 7.46 & 6.76 & 10.24 \\
\hline 120 & 0.305 & 0.008 & 0.679 & 2.49 & 1.92 & 3.35 & 7.24 & 4.19 & 8.65 \\
\hline 130 & 0.266 & 0.003 & 0.645 & 2.68 & 2.08 & 3.62 & 8.23 & 5.89 & 8.65 \\
\hline 140 & 0.083 & $<0.001$ & 0.451 & 2.92 & 2.31 & 3.78 & 8.67 & 9.76 & 8.76 \\
\hline 150 & 0.108 & $<0.001$ & 0.511 & 2.13 & 1.69 & 2.79 & 6.68 & 6.49 & 5.68 \\
\hline 160 & 0.123 & $<0.001$ & 0.523 & 2.65 & 2.07 & 3.44 & 10.48 & 6.30 & 6.37 \\
\hline 170 & 0.036 & $<0.001$ & 0.307 & 2.40 & 1.91 & 3.14 & 9.67 & 6.99 & 6.26 \\
\hline 180 & 0.049 & $<0.001$ & 0.363 & 2.58 & 2.07 & 3.45 & 9.87 & 5.43 & 8.72 \\
\hline 200 & 0.214 & 0.002 & 0.606 & 2.37 & 1.84 & 3.11 & 8.13 & 5.87 & 6.14 \\
\hline 220 & 0.199 & 0.002 & 0.577 & 1.88 & 1.44 & 2.49 & 5.25 & 4.67 & 6.43 \\
\hline 240 & 0.218 & 0.002 & 0.633 & 2.10 & 1.63 & 2.76 & 7.59 & 3.77 & 7.40 \\
\hline Cmax & 0.459 & 0.100 & 0.790 & 1.580 & 1.040 & 2.078 & 5.41 & 4.31 & 6.16 \\
\hline$\Delta \mathrm{Cmax}$ & 0.294 & 0.002 & 0.694 & 2.104 & 1.429 & 2.633 & 19.55 & 24.41 & 29.98 \\
\hline Tmax & 0.568 & 0.263 & 0.823 & 31.01 & 20.95 & 41.07 & 32.58 & 20.72 & 21.85 \\
\hline AUC & 0.293 & 0.001 & 0.636 & 347.7 & 244.7 & 423.9 & 26.66 & 25.92 & 33.84 \\
\hline
\end{tabular}

${ }^{1}$ Reliability analyses. Intraclass coefficient correlations (ranges from 0 to 1 ), typical error and coefficient of variation calculated for each time point across the three sodium bicarbonate supplementation sessions and for the time-to-peak blood bicarbonate (Tmax), peak blood bicarbonate $(\mathrm{Cmax})$ and maximal increase in blood bicarbonate $(\Delta \mathrm{Cmax})$ concentration. 
Table $2 .{ }^{1}$

\begin{tabular}{ccc}
\hline $\begin{array}{c}\text { Time after } \\
\text { ingestion (min) }\end{array}$ & $\begin{array}{c}\text { Probability of } \\
\text { increases above } \\
\mathbf{5} \mathbf{~} \mathbf{m o l} \cdot \mathbf{L}^{-\mathbf{1}}\end{array}$ & $\begin{array}{c}\text { Probability of } \\
\text { increases above } \\
\mathbf{6} \mathbf{~} \mathbf{m o l} \cdot \mathbf{L}^{\mathbf{1}}\end{array}$ \\
\hline 0 & $0 \%$ & $0 \%$ \\
15 & $0 \%$ & $0 \%$ \\
30 & $0 \%$ & $0 \%$ \\
45 & $8.6 \%$ & $0 \%$ \\
60 & $69 \%$ & $14 \%$ \\
75 & $93 \%$ & $60 \%$ \\
90 & $97 \%$ & $86 \%$ \\
105 & $99 \%$ & $93 \%$ \\
120 & $99 \%$ & $95 \%$ \\
135 & $99 \%$ & $96 \%$ \\
150 & $99 \%$ & $96 \%$ \\
165 & $99 \%$ & $95 \%$ \\
180 & $98 \%$ & $94 \%$ \\
195 & $97 \%$ & $92 \%$ \\
210 & $95 \%$ & $88 \%$ \\
225 & $91 \%$ & $80 \%$ \\
240 & $85 \%$ & $70 \%$ \\
\hline
\end{tabular}

${ }^{1}$ Probability estimates (\%) of elevating blood bicarbonate above $5 \mathrm{mmol} \cdot \mathrm{L}^{-1}$ and $6 \mathrm{mmol} \cdot \mathrm{L}^{-1}$ (from baseline) at different time points following sodium bicarbonate ingestion. Probability values were estimated using Bayesian simulation ( $\mathrm{n}=1$ million). 

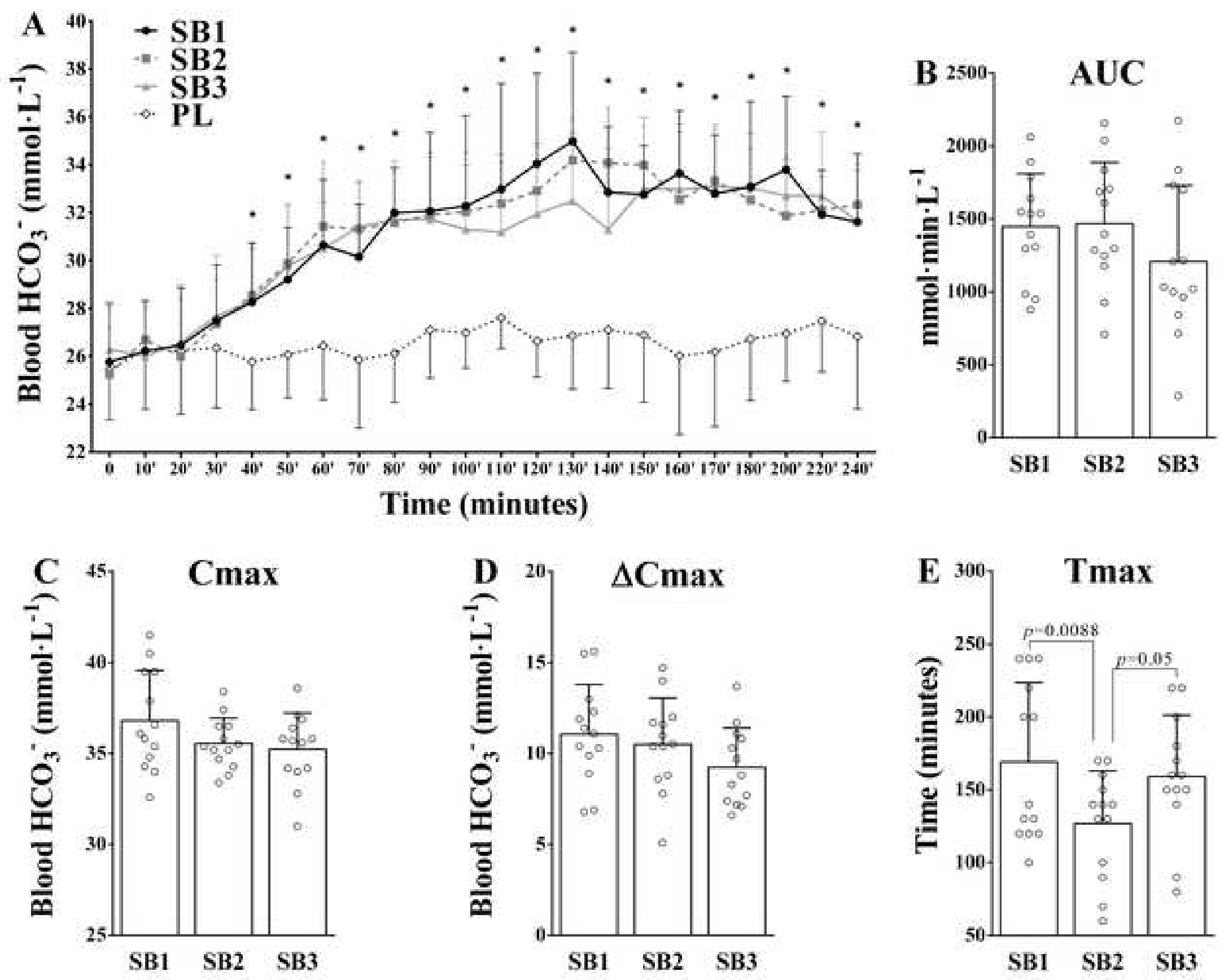
time after $\mathrm{SB}$ ingestion (min)

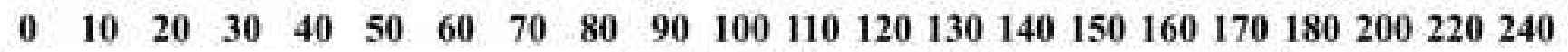

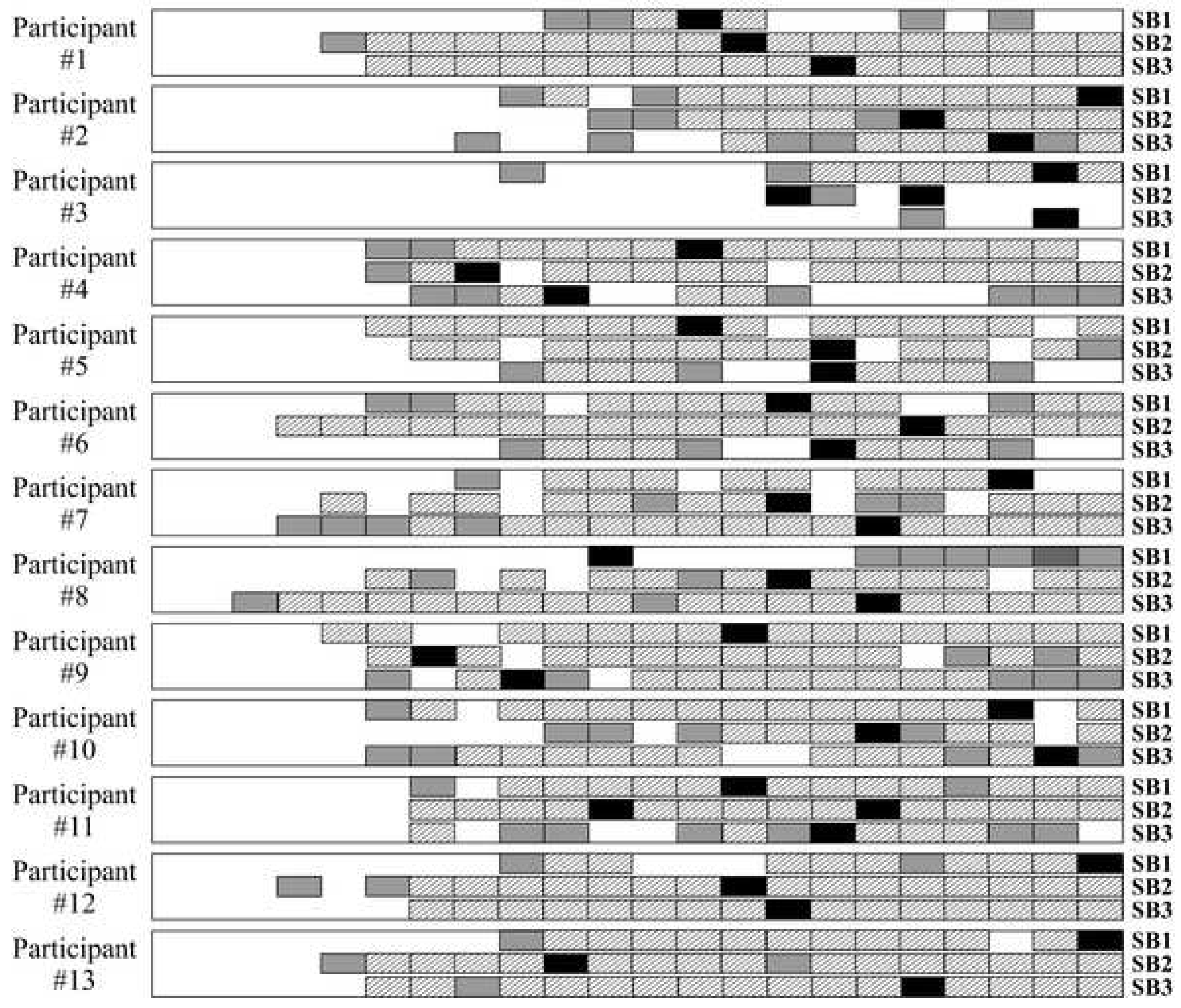




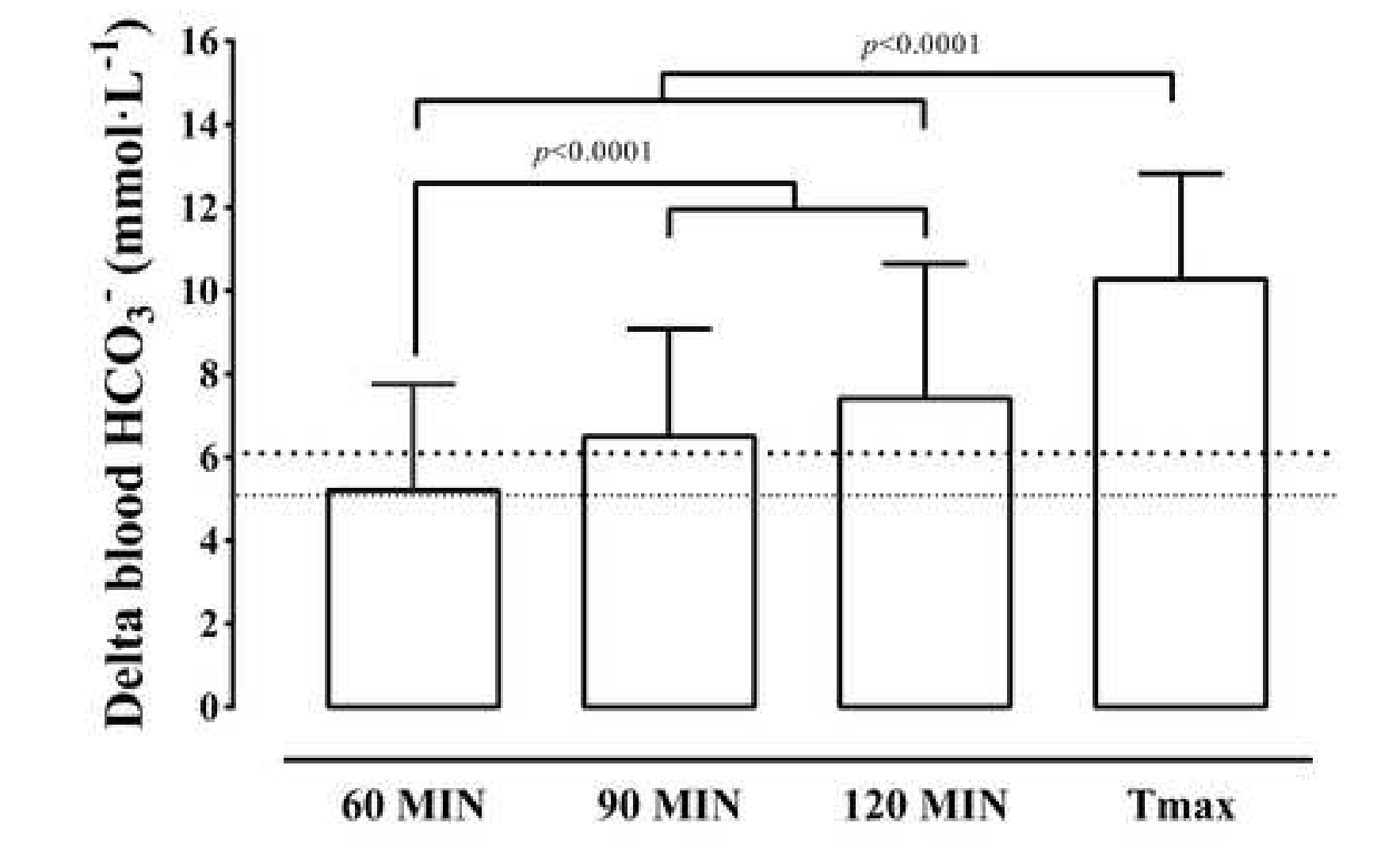

Figure 3

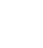

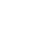

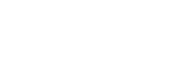

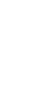

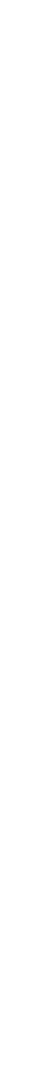




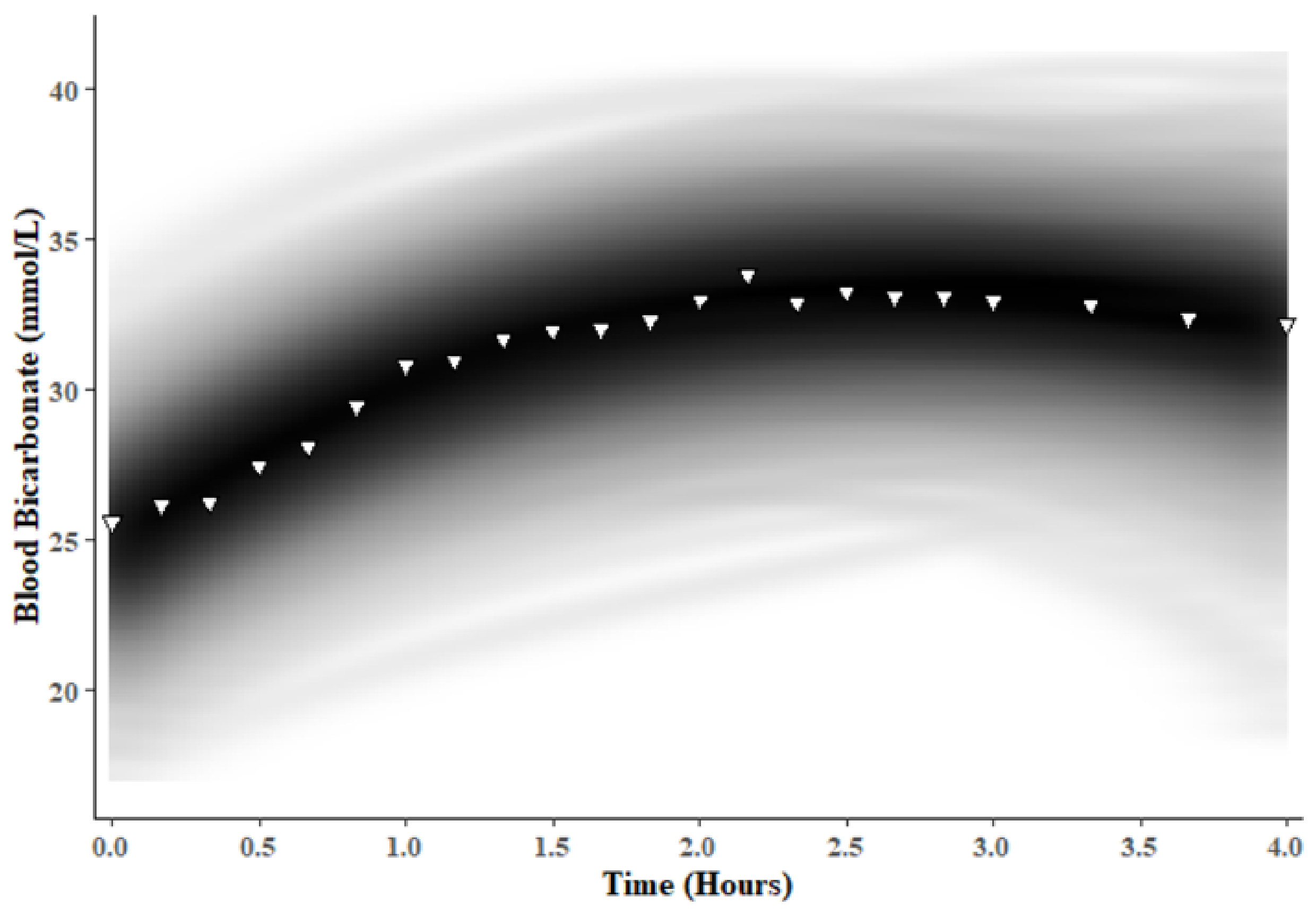



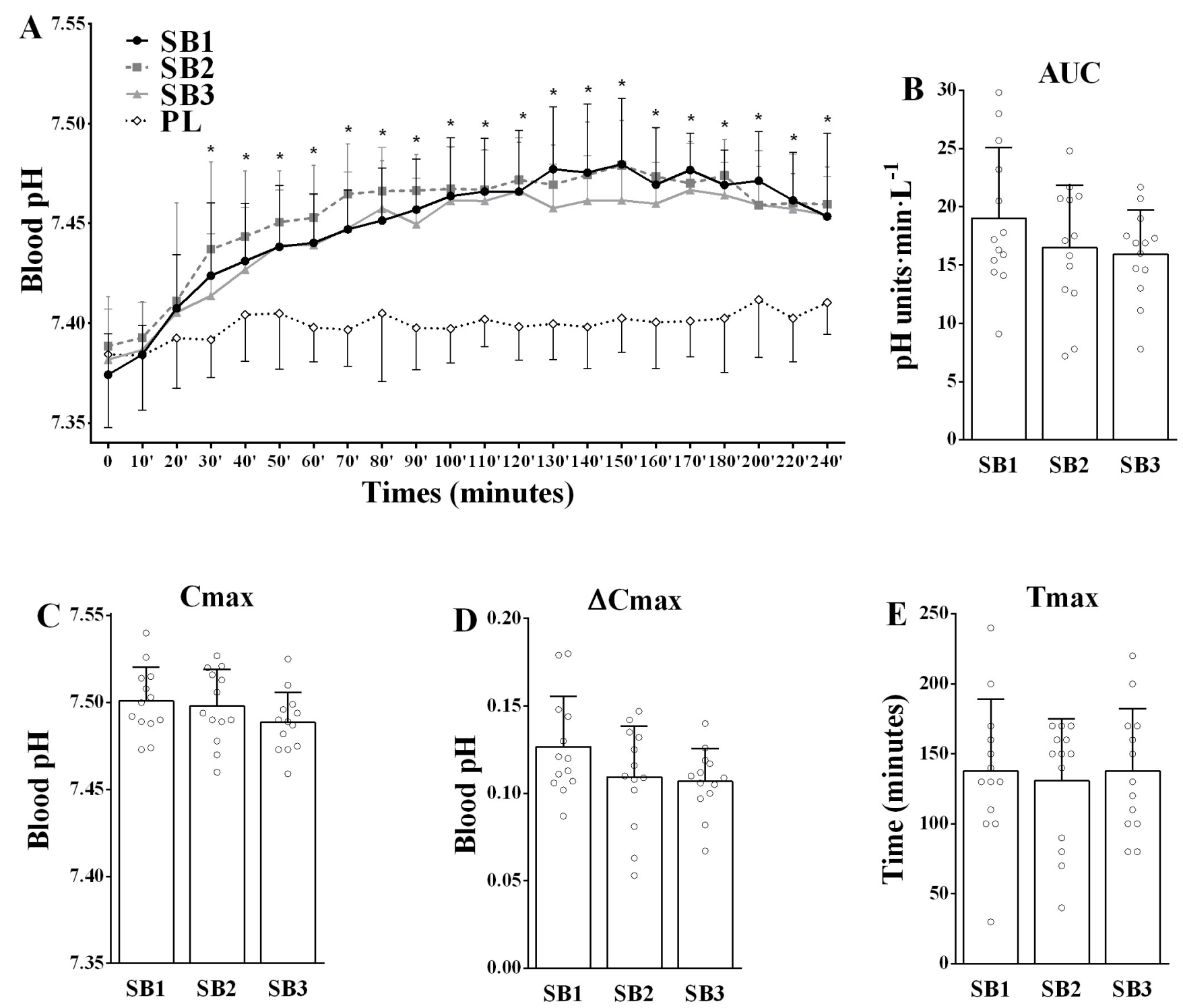


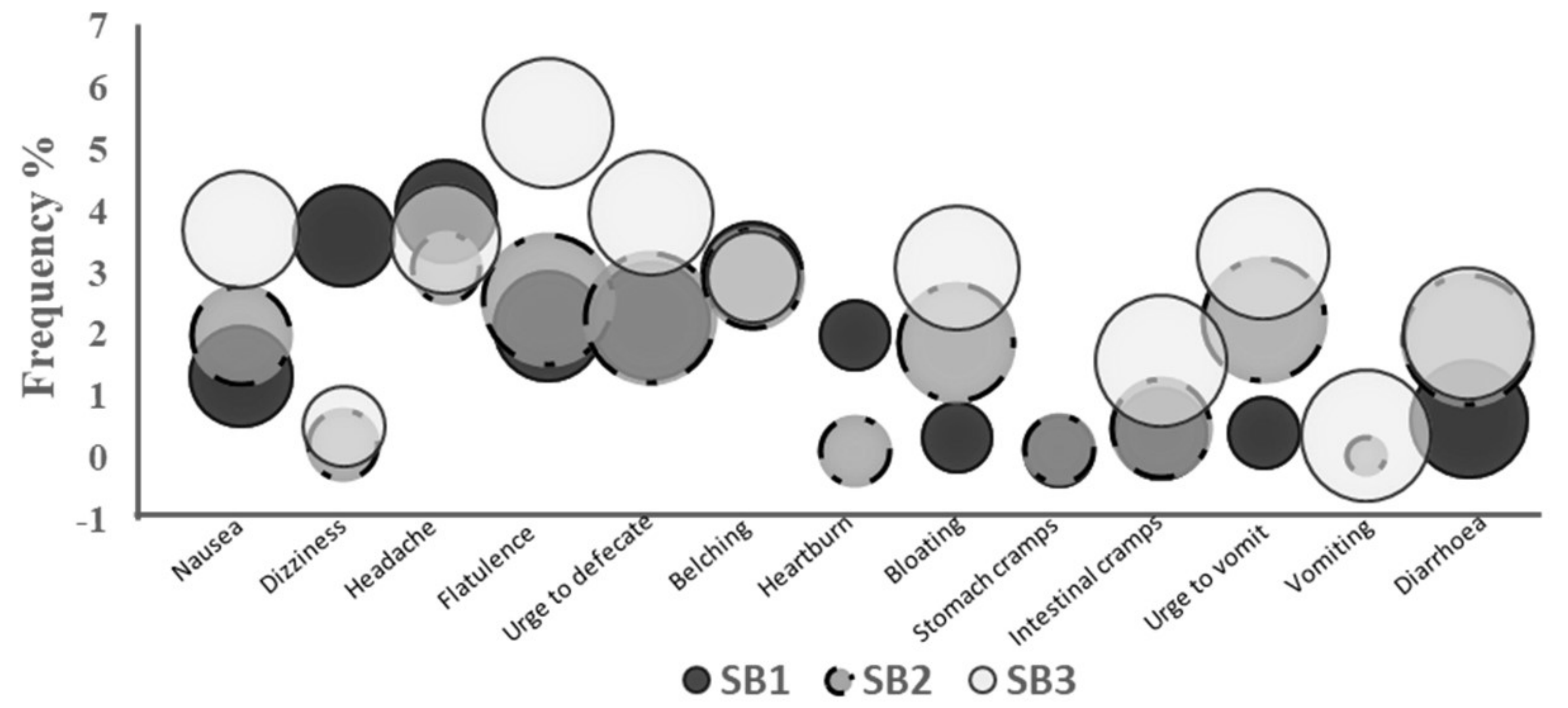

